\title{
Development of Automatically Updated Soundmaps for the Preservation of Natural Environment
}

\author{
Ioannis Paraskevas ${ }^{1}$, Stylianos M. Potirakis ${ }^{1}$, Ioannis Liaperdos ${ }^{2}$, Maria Rangoussi ${ }^{1}$ \\ ${ }^{1}$ Department of Electronics Engineering, Technological Education Institute of Piraeus, Aigaleo-Athens, Greece; ${ }^{2}$ Department of Tech- \\ nology of Informatics and Telecommunications, Technological Education Institute of Kalamata/Branch of Sparta, Sparta, Greece. \\ E-mail: iparaskevas@theiet.org, \{spoti, mariar\}@teipir.gr, gliaperd@teikal.gr
}

Received October $2^{\text {nd }}, 2011$; revised November $2^{\text {nd }}, 2011$; accepted December $3^{\text {rd }}, 2011$.

\begin{abstract}
Automatically Updated Soundmaps are maps that convey the sound rather than the visual information content of an area of interest, at a certain time instant or period. Sound features encapsulate information that can be combined with the visual features of the landscape, thus leading to useful environmental conclusions. This work aims to construct an Automatically Updated Soundmap of an area of environmental interest. A hierarchical pattern recognition approach method is proposed here that can exploit sound recordings collected by a network of microphones. Hence, after appropriate signal processing, the large amounts of information, originally in the raw form of sound recordings, can be presented in the concise yet meaningful form of a periodically updated soundmap.
\end{abstract}

Keywords: Soundmaps, Acoustic Ecology, Hierarchical Pattern Recognition, Network of Microphones

\section{Introduction}

Current research related to the environmental or ecological information of landscapes is mostly focused on their visual content, e.g., the landscape characteristics of a biotope. In this work, the sound content of the landscape is proposed as an additional information stream, aiming to produce useful audio-visual features, [1]. An Automatically Updated Soundmap (AUS) is the map of a certain region of environmental interest at a given time instant or period, which depicts the sound content, $[2,3]$. The periodic construction and comparison of AUSs for the same area is a useful tool for the detection of changes in an ecosystem, [4-6].

To this end, a method is proposed here for the development of AUSs for an area of environmental interest. In brief, the proposed method is based on sound recordings that are collected by microphones. Each sound recording is then processed and automatically classified. Classification results are placed on the exact recording spot of the geographical map of the area. The classification scheme proceeds hierarchically from coarser to finer decisions and categorization.

\section{Development of an AUS}

The proposed method for the development of an AUS is presented here based on a simulated recording setup.

\subsection{Microphone Placement for Optimal Area Coverage}

In order to develop an AUS, the sound content of a geographical area has to be recorded. A network of microphones deployed at appropriate spacing, can provide satisfactory spatial sampling. Spots of environmental interest are "sampled" more densely, whereas spots of lower interest and/or of restricted accessibility are sampled more sparsely. Eventual, sensors' (microphones) positioning is a compromise between mathematical optimality and practical restrictions.

Sound is recorded locally but is processed centrally. Specifically, a wireless sensor network is employed so as to communicate the pre-processed sound information from the sensors to the processing node (Figure 1).

\subsection{Pattern Recognition of Environmental Sounds}

The sound information gathered in the processing node is subsequently transmitted to the central point of the network (PC, in Figure 1), where the pattern recognition and the soundmap development steps take place. The pattern re- 


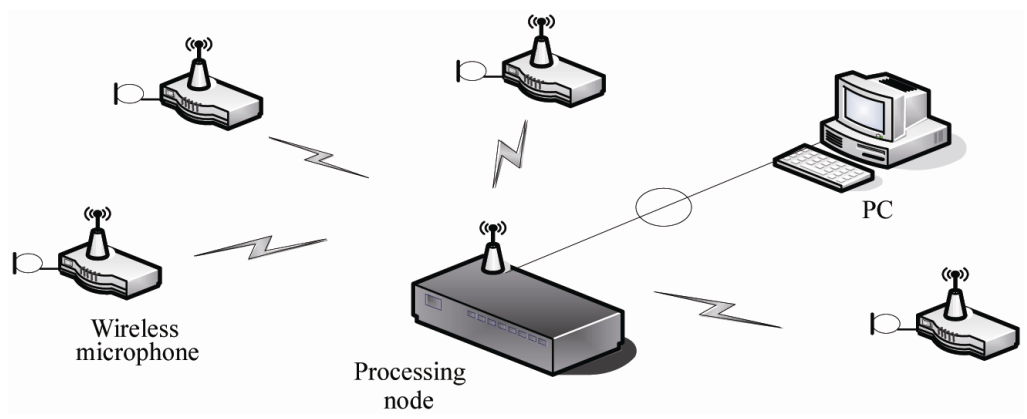

Figure 1. Wireless network of recording microphone.

cognition step includes i) the feature extraction and the ii) the classification stage, [7]. In feature extraction, class discriminating features are extracted in order to classify each sound recording to the corresponding sound class, $[8,9]$.

Pattern recognition of environmental sounds is a hierarchical process, [10]. Three main classes of environmental sounds are sought at a first (coarse) classification step, namely, anthropogenic, biophysical (other than anthropogenic) and geophysical sounds. Finer classification within each top-level class follows, e.g., classification of a biophysical sound into a certain species (e.g., bird, second level) and further into one of a finite number of members of this species (third level), [11,12].

All three classification steps aim to discriminate environmental sounds (in case they occur simultaneously) and

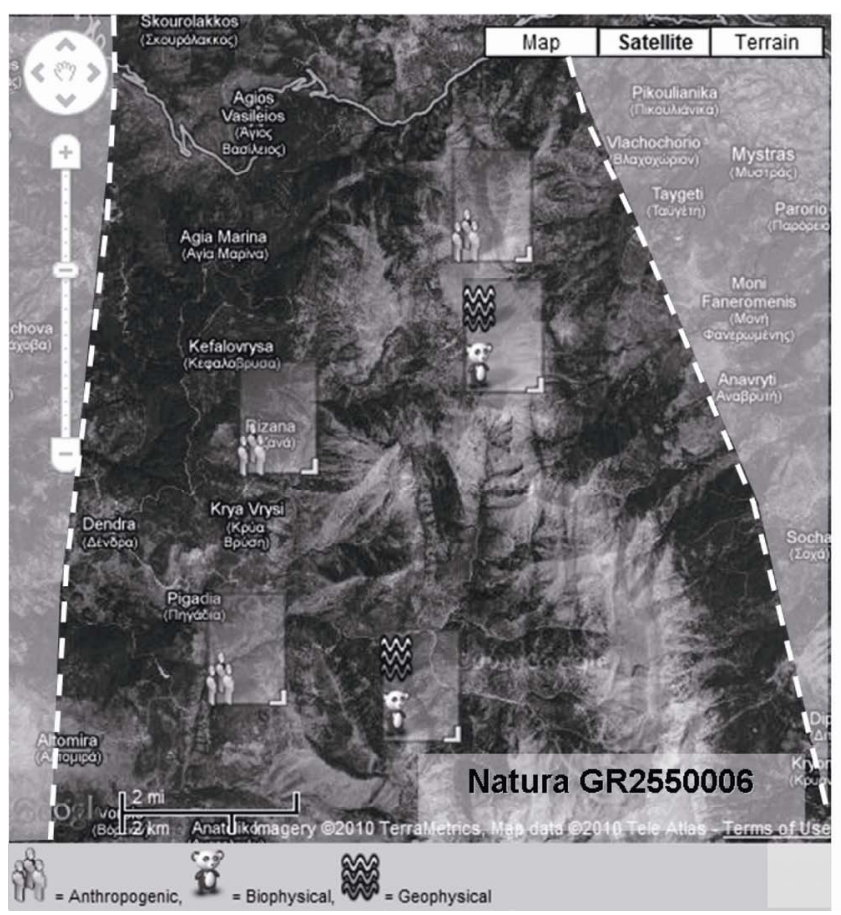

(a) to assign each sound to a certain class via algorithms for automatic pattern recognition. The classification of environmental sounds that convey similar time domain and frequency domain features, requires more sophisticated pattern recognition algorithms and therefore, the two finer classification steps are more demanding compared to the coarse classification step, [10].

\subsection{Soundmap Development}

An AUS may cover a wide area of environmental interest (e.g. a NATURA 2000 protected area, [13]) visualizing the results of automatic sound classification, as shown in

\section{Figure 2.}

Sound event classification results are stored in a data base in the central point (pc), in the format of Table 1. the term "event_id” (Table 1) stands for “event identifi-

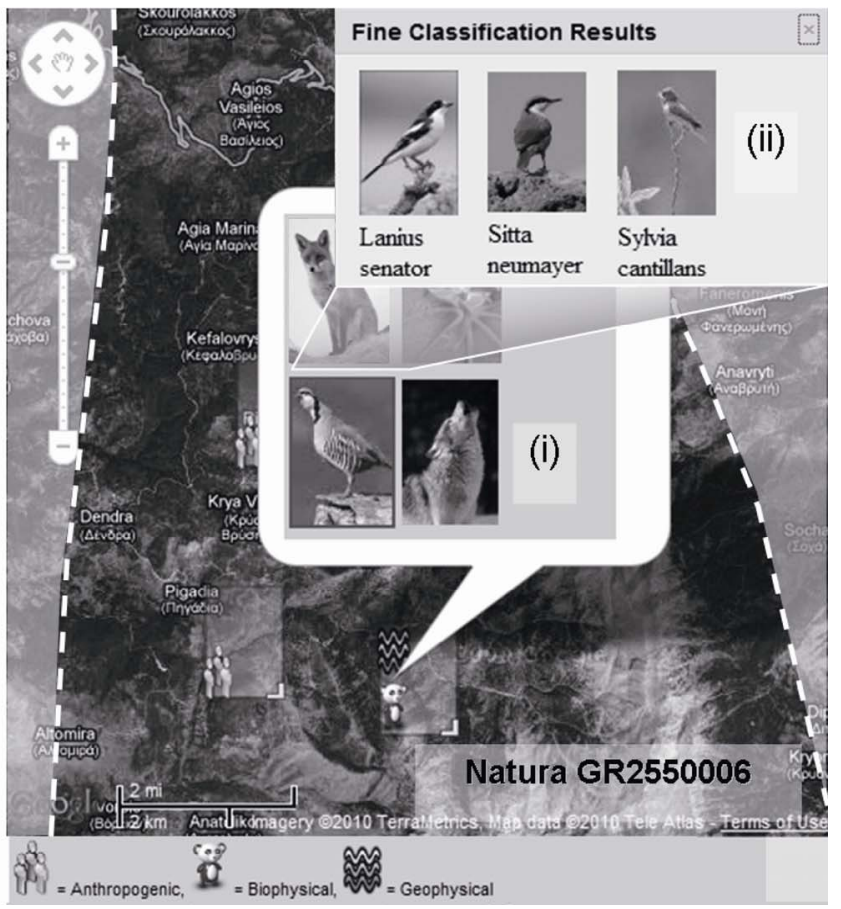

(b)

Figure 2. (a) Top-level classification, (b) second (i) and third (ii) classification levels, respectively. 
Table 1. Sound event.

Event_id Microphone_id Time stamp (date, time) Level 1: Coarse classification Level 2: Intermediate classification Level 3: Fine classification

$1423 \quad 4 \quad$ 05-15-2010, 19:35 Biophysical $\quad$ Bird $\quad$ Lanius senator

cation number" which is a unique number that corresponds to a specific sound event appearing in the "list of sound events" of the database. Microphone_id (Table 1) stands for "microphone identification number" and represents the number given to each microphone. The "microphone identification number" indicates the exact geographical area where a sound event is recorded; the geographical area is derived from the coordinates where the microphone has been placed.

The sound events are retrieved from the database in order to form the hierarchical soundmap, which includes three levels:

- $\quad$ The first, top-level presents the coarse classification results, by means of three markers, for the three aforementioned general classes of environmental sounds (anthropogenic, biophysical and geophysical, see Figure 2(a)). Note that, the location of the markers on the map correspond to the spot where the relative recording was taken.

- $\quad$ The second level presents the intermediate classification results, within the three main classes of the first level, e.g., species of fox, frog, bird and wolf within the class of biophysical sounds, (see Figure 2(b)-i).

- $\quad$ Finally, the third level presents the fine classification results, e.g., specific bird (here a Lanius senator) within the intermediate class of birds, see Figure 2(b)-ii.

\section{Conclusions}

Monitoring through the development and periodic update of soundmaps is a tool of practical interest for environmental surveillance of sensitive areas, e.g., regions of the NATURA 2000 network. A method for the development of AUSs for such areas is proposed and tested on a simulated environmental setup, with encouraging results. Further experimentation and adaptation with real field data is necessary before an efficient implementation is available.

\section{Acknowledgements}

Research co-funded by the EU (European Social Fund) and national funds, action "Archimedes III-Funding of research groups in T.E.I.”, under the Operational Programme "Education and Lifelong Learning 2007-2013".

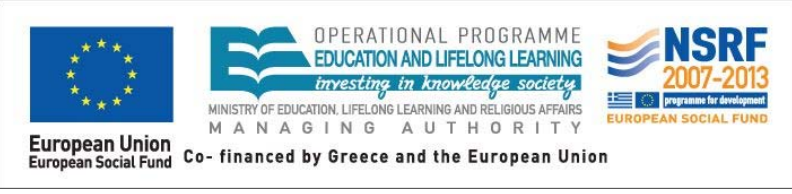

\section{REFERENCES}

[1] A. D. Mazaris, A. S. Kallimanis, G. Hatzigiannidis, K. Papadimitriou and J. D. Pantis, "Spatiotemporal Analysis of an Acoustic Environment: Interactions between Landscape Features and Sound,” Landscape Ecology, Vol. 24, No. 6, 2009, pp. 817-831. doi:10.1007/s10980-009-9360-x

[2] B. Krause, "Bioacoustics, Habitat Ambience in Ecological Balance,” Whole Earth Review, Vol. 57, 1987, pp. 267-271.

[3] B. Krause, "Wild Soundscapes: Discovering the Voice of the Natural World,” Wilderness Press, Berkeley, 2002.

[4] R. M. Schafer, "The Soundscape: Our Sonic Environment and the Tuning of the World,” Destiny Books, Rochester, 1993.

[5] M. G. Turner, R. H. Gardner and R. V. O’Neill, "Landscape Ecology in Theory and Practice: Pattern and Process,” Springer-Verlag, New York, 2001.

[6] SEKI Group, "Measurement and Analysis of Environmental Acoustics in Sequoia National Park: A Soundscape Perspective,” 2010.

http://envirosonic.cevl.msu.edu/seki

[7] R. O. Duda, P. E. Hart and D. G. Stork, "Pattern Classification,” 2nd Edition, John Wiley \& Sons, Ltd., Hoboken, 2000.

[8] E. Wold, T. Blum, D. Keislar and J. Wheaton, "Contentbased Classification, Search and Retrieval of Audio,” IEEE Multimedia, Vol. 3, No. 3, 1996, pp. 27-36. doi:10.1109/93.556537

[9] T. Zhang and C. C. J. Kuo, “Audio Content Analysis for Online Audiovisual Data Segmentation and Classification," IEEE Transactions on Speech and Audio Processing, Vol. 9, No. 4, 2001, pp. 441-457. doi:10.1109/89.917689

[10] I. Paraskevas, S. M. Potirakis and M. Rangoussi, "Natural Soundscapes and Identification of Environmental Sounds: A Pattern Recognition Approach," 16th International Conference on Digital Signal Processing (DSP'09), Santorini, 5-7 July 2009, pp. 1-6.

[11] I. Paraskevas and E. Chilton, "Combination of Magnitude and Phase Statistical Features for Audio Classification," Acoustics Research Letters Online, Vol. 5, No. 3, 2004, pp. 111-117. doi:10.1121/1.1755731 
[12] S. Parsons and G. Jones, "Acoustic Identification of Twelve Species of Echolocating Bat by Discriminant Function Analysis and Artificial Neural Networks," The Journal of Experimental Biology, Vol. 203, No. 17, 2000, pp. 2641-
2656.

[13] Natura, “2000 Ecological Network,” 2010. http://www.natura.org/ 